\title{
EDITORIAIS
}

\section{Litotripsia extracorpórea no tratamento de cálculos urinários em crianças}

\author{
Extracorporeal shock wave lithotripsy in the treatment of children's renal calculi
}

Eduardo de Almeida Rego Filho*

\begin{abstract}
A calculose urinária é doença muito comum em países industrializados, com prevalência entre 0,5 a $10 \%$, pico de incidência na $3^{\mathrm{a}}$ e $4^{\mathrm{a}}$ décadas de vida, e predomínio nos homens, em proporção de quatro para uma mulher. O risco de recorrência é alto, ao redor de $50 \%$ em cinco anos ${ }^{1}$. Não há dados sobre prevalência em nosso meio ${ }^{2}$.

A sintomatologia da litíase urinária em crianças é bastante variada. Observa-se dor abdominal recorrente, hematúria, disúria, polaciúria e, mais raramente, cólica renal típica e incontinência urinária. A forma assintomática, freqüentemente um achado durante investigação de hematúria microscópica monossintomática, e a ocorrência familiar, são significativas.

Durante muitos anos, a litíase renal em crianças foi considerada um achado muito raro. Desde a introdução dos modernos estudos por imagem (ultra-sonografia, tomografia e ressonância magnética), a calculose tem sido descoberta com maior freqüência e em idades mais precoces. Dados recentes de literatura indicam que aproximadamente $7 \%$ dos cálculos ocorrem em crianças e adolescentes até os 16 anos $^{3}$.

Duas outras grandes mudanças foram observadas nos últimos 20 anos. A primeira foi o aumento da capacidade de determinar o fator ou os fatores responsáveis pela formação do cálculo. Na maioria dos centros especializados, a investigação sistematizada de sua etiologia permite a identificação da(s) causa(s) em mais de $80 \%$ dos pacientes ${ }^{4}$.

A hipercalciúria idiopática normocalcêmica está presente como fator etiológico principal da formação calculosa em 40 a $50 \%$ dos casos. Apresenta padrão de ocorrência

\footnotetext{
* Professor Titular de Pediatria. Doutor em Medicina. Departamento de Pediatria e Cirurgia Pediátrica da Universidade Estadual de Londrina, Paraná. Pediatra e Nefrologista. Responsável pelo Setor de Nefrologia
} Pediátrica do Hospital Universitário Regional do Norte do Paraná.
\end{abstract}

familiar, com comprometimento de parentes em número muito acima do observado na população geral ${ }^{5}$.

A segunda mudança observada refere-se ao tratamento da calculose urinária. Até 1980, a conduta médica se restringia ao atendimento de urgência, alívio da dor, aumento do aporte de líquidos, controle da infecção quando presente e, em alguns casos, no preparo dos pacientes para tratamento cirúrgico aberto.

Hoje, a litotripsia extracorpórea por ondas de choque (LECO), a nefrolitotripsia percutânea (NLPC) e a ureterorenolitotripsia endoscópica mudaram substantivamente a eficácia e a segurança no tratamento dos pacientes. São consideradas técnicas "minimamente invasivas", e sua utilização em crianças é consagrada ${ }^{6-9}$. A cirurgia aberta passou a ser necessária em menos de $10 \%$ dos casos.

A publicação do artigo "A litotripsia extracorpórea no tratamento de cálculos em crianças" do Prof. Duarte, vem ao encontro de duas necessidades relacionadas: intensificar os conhecimentos dos pediatras sobre a técnica, e corrigir uma lacuna no Jornal de Pediatria, que não abordou o tema nos últimos dez anos.

A pesquisa deve ser considerada um trabalho retrospectivo de estudo de série de casos sem grupo controle e com intervenção analisada "antes e depois". Não oferecendo maiores detalhes sobre a seleção de pacientes, sobre a indicação da litotripsia e sobre o protocolo de busca sistematizada para detecção de complicações além das citadas (infecciosas, hemorrágicas e obstrutivas), assim como ausência de tratamento estatístico, além do estritamente descritivo, os autores dificultam comparações com dados de outros centros ou mesmo generalização dos resultados. Por outro lado, a origem do trabalho, o número de pacientes e os resultados apresentados, que são semelhantes aos observados na literatura, permitem que esta publicação atinja seus objetivos. 
Em uma criança, a remoção de um cálculo depende da sua composição, localização e tamanho, da presença de obstrução, de infecção, ou de ambas ${ }^{3}$. A LECO é, na maioria dos casos, a primeira opção de tratamento para a remoção de cálculo ${ }^{6}$. O método baseia-se na emissão de ondas acústicas, que geram um súbito gradiente de pressão em um pequeno espaço físico. As ondas produzidas e emitidas por uma fonte energética são convergentes e se concentram sobre um foco (cálculo), que é localizado por fluoroscopia ou ultra-sonografia. Quando a onda de choque atinge o cálculo e supera sua força de coesão, há a fragmentação, com mínima lesão no tecido circunvizinho.

O sucesso no tratamento é caracterizado quando o paciente, após um período de observação de três meses, torna-se livre do cálculo ou mantém cálculo menor que $4 \mathrm{~mm}$, estando assintomático. A eficácia do tratamento deve considerar, ainda, a necessidade de retratamento e a freqüência de complicações.

Nos anos 80 , vários estudos clínicos e experimentais comprovaram inúmeras complicações deste tratamento (dor em queimação no local, lesão pulmonar, hematúria macroscópica, hematomas renais, necrose tubular, sepse, hipertensão arterial, obstrução ureteral e redução de função renal), que produziram uma situação fundamentada de apreensão entre os pediatras. Com o aumento da experiência dos profissionais, o lançamento no mercado de equipamentos de nova geração e as modificações na técnica de aplicação, principalmente quanto ao número de ondas de choque $\mathrm{e}$ quantidade de energia liberada, observou-se mudança na aceitação do tratamento, que hoje deve ser considerada segura, eficaz, minimamente invasiva, e com baixos índices de morbidade.

Mesmo com os resultados alentadores sobre a segurança da técnica, persistem as publicações detectando alterações funcionais transitórias ${ }^{10}$. Esses fatos sugerem a necessidade de manter o controle da freqüência, intensidade e transitoriedades dos possíveis efeitos colaterais indesejáveis da litotripsia.

Devemos, também, lembrar que a litotripsia extracorpórea por ondas de choque, em alguns casos, pode complementar ou ser complementada por outras técnicas de remoção do cálculo ${ }^{11}$.

Alguns estudos vêm indicando vantagens na utilização da LECO em crianças, quando comparadas com o adulto: o tecido da criança, com maior teor de líquido, facilita a propagação da energia; a compactação do cálculo (idade do cálculo) é menor na infância, e a eliminação dos fragmentos causam menor dificuldade na criança pequena do que em crianças maiores e adultos ${ }^{12}$. Estudos realizados na França (Hopital Saint Joseh), em 19 crianças com idade entre 5 e 24 meses, observaram resultados excelentes com a LECO - dez crianças ficaram livres de cálculo com uma sessão, e oito com duas sessões de tratamento; não foram observadas complicações do tratamento ${ }^{13}$.

O trabalho do Prof. Duarte e os dados de literatura nos indicam que os melhores resultados são observados em pacientes com cálculos menores que $20 \mathrm{~mm}$, localizados na pelve renal, nos cálices superiores e médios e no ureter. Os cálculos maiores, em grupo caliceal inferior, e a presença de anomalias anatômicas, em geral se associam aos piores índices de sucesso.

Os resultados dos estudos modernos sobre calculose em pediatria permitem concluir que:

- o diagnóstico de litíase do trato urinário está se tornando mais freqüente, inclusive em crianças de baixa idade (recém-nascidos e prematuros);

- a capacidade de determinar o fator ou os fatores responsáveis pela formação do cálculo é alta;

- a hipercalciúria idiopática, isoladamente ou em associação com outras desordens metabólicas, as malformações de vias urinárias e as infecções são responsáveis pela maioria dos casos de litíase;

- a litotripsia extracorpórea com ondas de choque tornouse a primeira opção para remoção da maioria dos cálculos, com nível significativo de segurança e eficácia.

\section{Referências bibliográficas}

1. Monk RD. Clinical approach to adults. Semin Nephrol 1996; 19:889-907.

2. Laranja SMR, Hielberg IP, Coelho STSN, Novoa CG, Schor N. Estudo multicêntrico da litíase renal do Brasil (Multilit). In: Schor N, Hielberg IP, eds. Calculose renal. São Paulo: Sarvier; 1995. p.295-8.

3. Elder SJ. Litíase urinária. In: Behrman RE, Kliegman RM, Jenson $\mathrm{HB}$, editores. Tratado de Pediatria. $16^{\mathrm{a}}$ ed. Rio de Janeiro: Guanabara Koogan; 2002.p.1630-3.

4. Rocha RCG. Avaliação metabólica em pacientes portadores de nefrolitíase e controles em Londrina - Paraná [dissertação]. Londrina: Universidade Estadual de Londrina; 2001.

5. Obregón JMV. Estudo familiar da hipercalciúria idiopática [dissertação]. Londrina: Universidade Estadual de Londrina; 2000.

6. Netto NR Jr.. Litíase urinária. In: Netto NR Jr., editor. Urologia Prática. $4^{\mathrm{a}}$ ed. São Paulo: Atheneu; 1999.p.61-80.

7. Brinkmann AO, Griehl A, Kuwertz-Broking E, Bulla M, Hertle L. Extracorporeal shock wave lithotripsy in children. Efficacy, complications and long-term follow-up. Eur Urol 2001;39:591-7.

8. Shukla AP, Hoover DL, Homsy YL, Perlman S, Shurman S, Reisman EM. Urolithiasis in the low birth weight infant: the role and efficacy of extracorporeal shock wave lithotripsy. J Urol 2001;165:2320-3

9. Elsobky E, Sheir KZ, Madbouly K, Mokhtar AA. Extracorporeal shock wave lithotripsy in children: experience using two secondgeneration lithotripters. BJU Int 2000;86:851-6.

10. Villanyi KK, Szekely JG, Farkas LM, Javor E, Pusztai C. Shortterm changes in renal function after extracorporeal shock wave lithotripsy in children. J Urol 2001;166:222-4.

11. Choong S, Whitfield H, Duffy P, Kellett M, Cuckow P, Van't Hoff $\mathrm{W}$, et al. The management of paediatric urolithiasis. BJU Int 2000; 86:857-60.

12. Gofrit ON, Pode D, Meretyk S, Katz G, Shapiro A, Golijanin D, et al. Is the pediatric ureter as efficient as the adult ureter in transporting fragments following extracorporeal shock wave lithotripsy for renal calculi larger than $10 \mathrm{~mm}$ ? J Urol 2001; 166:1862-4.

13. Lottmann HB, Archambaud F, Traxer O, Mercier-Pageyral B, Helal B. The efficacy and parenchymal consequences of extracorporeal shock wave lithotripsy in infants. BJU Int 2000; 85:311-5. 